\title{
An Electrochemical Immunosensor for Rapid and Sensitive Detection of Mycotoxins Fumonisin B1 and Deoxynivalenol
}

\author{
$\operatorname{Lin} \mathrm{Lu}^{1}$ \\ Rajesh Seenivasan ${ }^{1}$ \\ Yi-Cheng Wang ${ }^{1}$ \\ Jae-Hyuk $\mathrm{Yu}^{2}$ \\ and \\ Sundaram Gunasekaran ${ }^{1} *$ \\ ${ }^{1}$ Department of Biological Systems Engineering \\ ${ }^{2}$ Department of Bacteriology \\ University of Wisconsin-Madison \\ Madison, WI 53706, USA
}

* Corresponding author e-mail: guna@wisc.edu 


\begin{abstract}
We report an electrochemical immunosensing method for rapid and sensitive detection of two mycotoxins, fumonisin B1 (FB1) and deoxynivalenol (DON). A disposable screen-printed carbon electrode (SPE) was used as sensing platform. The working electrode part of SPE was modified by gold nanoparticles (AuNPs) and polypyrrole (PPy)electrochemically reduced graphene oxide (ErGO) nanocomposite film for effective antitoxin antibody immobilization, enhanced electrical conductivity, and biocompatibility. Under optimized test conditions, the limit of detection and linear range achieved for FB1 were 4.2 $\mathrm{ppb}$ and 0.2 to $4.5 \mathrm{ppm}(\% \mathrm{RSD}=4.9 \%)$; and the corresponding values for DON were $8.6 \mathrm{ppb}$ and 0.05 to $1 \mathrm{ppm}(\% \mathrm{RSD}=5.7 \%)$. The immunosensor can specifically detect the target toxin in co-existing toxins environment. The sensor exhibited high sensitivity and low matrix interference when tested using extracts obtained from spiked corn samples. Hence, our electrochemical immunosensing scheme can be adopted for highly sensitive and rapid detection of multiple co-contaminant mycotoxins in food and feed products.
\end{abstract}

\title{
Keywords
}

DON, electrochemical immunosensing, fumonisin, mycotoxins, reduced graphene oxide 


\section{INTRODUCTION}

Mycotoxins such as aflatoxin (AF), deoxynivalenol (DON), fumonisin B1 (FB1), ochratoxin A (OTA) and zearalenone are a group of toxic secondary metabolites produced by certain fungi. They naturally contaminate foods and feeds [1], which lower the product quality, pose severe health risk to humans and animals, and cause profound economic losses worldwide [2]. FB1 is the most common and economically important form of fumonisin (FUM), given its hepatotoxic and nephrotoxic effects in all animal species tested [2,3]. DON occurs predominantly in grains such as wheat and barley infected by Fusarium head blight or scab $[4,5]$. The US Food and Drug Administration has suggested action levels of 2 to 4 parts per million (ppm) for FUM and $1 \mathrm{ppm}$ for DON in foods intended for human consumption [6]. In addition, the co-occurrence of mycotoxins in nature has increased their likelihood of consumption and may cause additive and/or synergistic effects [7, 8]. For example, AF and FUM are co-contaminants often found in corn [9-11] and milled corn fractions [5, 9]; DON and OTA are common co-contaminants in wheat $[4,12,13]$.

Due to the widespread prevalence of multiple mycotoxins in foods and feeds, research has focused on developing effective methods for highly sensitive and selective detection of mycotoxins to screen foods and feeds. Traditional analytical methods include liquid chromatography-mass spectrometry (LC-MS), high performance liquid chromatography (HPLC), and thin-layer chromatography (TLC), which can detect toxins in the range of $\sim 0.01$ to 0.1 parts per billion (ppb) [14-18]. However, these laboratory methods are not suitable for rapid, on-site testing because they are labor- and time-intensive as well as expensive [19]. Other screening methods used for mycotoxins detection are enzyme-linked immunosorbent assay (ELISA), surface plasmon resonance (SPR), lateral flow immunoarray (LFI), immunochip, electronic nose, etc. [18]. Though ELISA is widely used, its shortcomings include cross-reactivity and possible false-positive or false-negative results [18]; also, 
additional reagents are necessary to ensure the stability of stored antibody ( $\mathrm{Ab}$ ) coating on the microwell plates [20]. SPR sensor is sensitive to temperature, and LFI and electronic nose have low sensitivity, while immunochip involves complex labeling process and requires professional technicians to run the test [18].

Electrochemical biosensors are attractive due to their advantages of operational simplicity, high sensitivity, low cost, and portable on-field use. Kadir and Tothill developed the ELISA-based chronoamperometric immunosensor using screen-printed gold electrode for detection of FUM with a limit of detection (LOD) of $5 \mu \mathrm{g} / \mathrm{L}$ (5 ppb) [21]. However, the use of secondary $\mathrm{Ab}$ in this work increases the cost and complexity. Ezquerra et al. reported an electrochemical immunoassay with the help of dispersed paramagnetic particles for FB1 detection with LOD of $0.58 \pm 0.05 \mu \mathrm{g} / \mathrm{L}$ [22]. But this method requires additional tracer as well as an external magnetic field, also involves a step to transfer modified magnetic beads to the electrode surface, which is somewhat complicated.

Novel nanomaterials are widely used for effective Ab immobilization and highly sensitive biosensor development. Srivastava et al. synthesized and deposited chemically active reduced graphene oxide (rGO) onto an indium tin oxide-coated glass substrate and this platform has been utilized for covalent attachment of the AFB1 antibodies for AFB1 detection $(\mathrm{LOD}=0.12 \mathrm{ng} / \mathrm{mL})$ [23]. Romanazzo et al. developed a recombinant fragment antigen-binding fragment based electrochemical immunosensor for DON detection [24]. Zhilei et al. reported a sensor for using fullerene C-60, an ionic liquid and ferrocene on a chitosan film at a glassy carbon electrode to detect DON [25]. Some literature report that incorporating nanomaterials and conducting polymers together in sensor platform afford large surface area, biocompatibility, ease of functionalization, and significantly enhances the sensitivity, reproducibility, and stability [26, 27]. Among nanomaterials, graphene oxide (GO)/polypyrrole (PPy) nanocomposite, electrochemically reduced graphene oxide (ErGO) 
and gold nanoparticles (AuNPs) are particularly suitable for biosensor applications due to their high electrical conductivity, good biocompatibility and chemical stability [28-33]. PPy is one of the most studied conducting polymers due to its great electrical conductivity, ease of preparation and low cost. However, it has some drawbacks such as poor mechanical strength and instability in conductivity [33]. Researchers have discovered that GO demonstrates a synergistic effect in PPy/GO composites that could improve both mechanical properties and electrical conductivity compared to PPy [33]. Therefore, combining such advantages of conducting polymers and nanomaterials [26, 34], we developed a label-free electrochemical immunosensing method using disposable screen-printed carbon electrode (SPE) modified by AuNPs and PPy/ErGO film for effective Ab immobilization and improved electrical conductivity to enable highly sensitive and selective detection of FB1 and DON.

\section{EXPERIMENTAL}

\subsection{Materials and chemicals}

SPEs were purchased from CH Instruments, Inc. (TE100, Bee Cave, TX, USA). Graphite flakes $(\sim 150 \mu \mathrm{m})$, FB1, DON and bovine serum albumin (BSA) were purchased from Sigma-Aldrich (St. Louis, MO, USA). Hydrogen tetrachloroaurate(III) trihydrate $\left(\mathrm{HAuCl}_{4} \cdot 3 \mathrm{H}_{2} \mathrm{O}\right)$, nitric acid $(63 \%)$, pyrrole $(99 \%$ extra pure) and 3-mercaptopropionic acid (MPA, 99+ \%) were supplied by ACROS Organics (Morris Plains, NJ, USA). Monoclonal anti-DON mouse $\mathrm{Ab}(1 \mathrm{mg} / \mathrm{mL})$ and monoclonal anti-FB1 mouse $\mathrm{Ab}(1 \mathrm{mg} / \mathrm{mL})$ were purchased from Antibodies-online.com (Atlanta, GA, USA). Immobilization of antibodies onto the electrode surface was performed using 1-ethyl-3-(3-dimethylaminopropyl) carbodiimide (EDC) and N-hydroxysuccinimide (NHS) chemistry with a minimum purity of $98 \%$ or higher (Bioworld, Dublin, OH, USA and Acros Organics). Phosphate buffered saline (PBS) 10X, methanol (99.8\%), sulfuric acid (96\%), hydrogen peroxide (50\%), and 
hydrochloric acid (36.5-38 \%) were from Fisher Scientific (Rockford, IL, USA). Potassium ferrocyanide trihydrate (reagent grade) and potassium chloride were acquired from Fisher Science Education (Hanover Park, IL, USA). Potassium ferricyanide, sodium phosphate monobasic $\left(\mathrm{NaH}_{2} \mathrm{PO}_{4}\right)$ and sodium phosphate dibasic $\left(\mathrm{Na}_{2} \mathrm{HPO}_{4}\right)$ were certified A.C.S. reagents from Thermo Fisher Scientific (Fair Lawn, NJ, USA). All chemicals were used as received without any purification, and deionized (DI) water of resistivity $\geq 18.2 \mathrm{M} \Omega \cdot \mathrm{cm}$ (Ultrapure water system, Millipore, Billerica, MA, USA) used for solution preparation and all experiments.

Methods for all solution preparation, synthesis of GO and AuNPs, and extraction of spiked toxins in corn samples are presented in Supplementary Material.

\subsection{Immunosensor fabrication}

The immunosensor fabrication steps in preparing the working electrode surface of SPE is illustrated in Scheme 1A. The SPE is first rinsed with DI water to remove any existing impurities. PPy/GO nanocomposite film, grown using our modified procedure [27], is electrochemically deposited over the bare SPE placed in nitrogen-purged solution containing $0.025 \mathrm{M}$ pyrrole, $0.33 \mathrm{mg} / \mathrm{mL} \mathrm{GO}$ and $0.1 \mathrm{M} \mathrm{KCl}$ under gentle stirring. Cyclic

voltammetry $(\mathrm{CV})$ scans are then applied between $-0.2 \mathrm{~V}$ and $+0.9 \mathrm{~V}$ for 15 complete cycles at $50 \mathrm{mV} / \mathrm{s}$ scan rate to electro-polymerized pyrrole into PPy and deposit PPy/GO nanocomposite film on the SPE, with GO being partially reduced during the process. GO is fully reduced and PPy/ErGO film is obtained by $\mathrm{CV}$ from 0.0 to $-1.4 \mathrm{~V}$ at $50 \mathrm{mV} / \mathrm{s}$ scan rate in nitrogen-purged $0.05 \mathrm{M}$ phosphate buffer $\left(\mathrm{Na}_{2} \mathrm{HPO}_{4} / \mathrm{NaH}_{2} \mathrm{PO}_{4}\right)$ solution ( $\left.\mathrm{pH} 7.0\right)$ for 25 complete cycles [35]; it is then rinsed with DI water and dried at room temperature. The ErGO/PPy film surface is drop-coated with $20 \mu \mathrm{L}$ AuNPs solution and allowed to dry under ambient conditions. The AuNPs-PPy/ErGO-modified SPE is immersed in $2 \mathrm{~mL}$ of $10 \mathrm{mM}$ 
MPA solution and incubated for $6 \mathrm{~h}$ at room temperature to form Au-S bond [36]. After rinsing with DI water, the MPA-functionalized electrode is transferred to a mixture of EDC/NHS as crosslinker and incubated for one hour at room temperature to activate the carboxyl groups for effective immobilization of antibodies on the electrode surface [37]. The electrode is washed three times with DI water to remove any excess EDC/NHS and byproducts followed by incubating with $40 \mu \mathrm{L}$ of anti-FB1 or anti-DON Ab solution ( $\mathrm{pH}$ 9.0) at $4{ }^{\circ} \mathrm{C}$ for $12 \mathrm{~h}$ to allow sufficient $\mathrm{Ab}$ immobilization. Finally, the electrode is incubated with 1\% BSA for 30 min to block unoccupied sites and washed with 1X PBS [38]. The fabricated Ab-AuNPs-PPy/ErGO-SPE is stored in $1 \mathrm{X}$ PBS (pH 7.4) at $4{ }^{\circ} \mathrm{C}$ when not in use.

\subsection{Instruments and measurements}

Fourier transform infrared spectrometer (FT-IR, Spectrum-100, PerkinElmer) was used for characterization of synthesized GO. UV/Vis spectrophotometer (Lambda-25, PerkinElmer) was also used to characterize the synthesized GO and AuNPs. All electrochemical experiments were performed in $\mathrm{CHI}-660 \mathrm{D}$ electrochemical workstation $(\mathrm{CH}$ Instruments, Inc. USA) with a conventional three-electrode system. CV and differential pulse voltammetry (DPV) of redox couple $5 \mathrm{mM}\left[\mathrm{Fe}(\mathrm{CN})_{6}\right]^{3-} /\left[\mathrm{Fe}(\mathrm{CN})_{6}\right]^{4-}$ containing $1 \mathrm{M} \mathrm{KCl}$ as supporting electrolyte was employed to investigate the electron transfer behavior of SPE after each modification step and for target toxins (FB1/DON) detection. Scanning electron microscopy (SEM) images were obtained using a LEO 1530 scanning electron microscope.

\section{RESULTS AND DISCUSSION}

The sensing scheme employed the target mycotoxin detection system is illustrated in Scheme 1B. With the target mycotoxin-specific Ab attached on the working surface of SPE, a blank sample is tested and the DPV peak current is recorded as the signal baseline. When 
samples containing mycotoxins are tested, after 40 min of incubation, the Ab-toxin interaction at the electrode surface results in reduced electrochemical current due to the insulating property of the Ab-toxin complex [39]. The decrease in DPV current is proportional to the Ab vs. target toxin concentration of the tested sample. Hence, the amount of target toxins present in the sample could be quantified based on the magnitude of the electrical signal obtained.

\subsection{FT-IR and UV-Vis characterization of synthesized GO}

FTIR spectrum of GO (Fig. S1a, Supplementary Material) revealed the characteristic $-\mathrm{OH}\left(\right.$ at $\left.3347 \mathrm{~cm}^{-1}\right), \mathrm{C}=\mathrm{O}\left(\right.$ at $\left.1729 \mathrm{~cm}^{-1}\right), \mathrm{C}=\mathrm{C}\left(\right.$ at $\left.1624 \mathrm{~cm}^{-1}\right)$, and $\mathrm{C}-\mathrm{O}\left(\right.$ at $\left.1071 \mathrm{~cm}^{-1}\right)$ bonds, which indicated the presence of oxygenated groups on GO [27, 40]. In the characteristic UV-vis spectrum of the as-synthesized GO suspension (Fig. S1b), the peak at $226 \mathrm{~nm}$ corresponds to the $\pi \rightarrow \pi^{*}$ transitions of the aromatic $\mathrm{C}=\mathrm{C}$ bond, and a shoulder at $\sim 300 \mathrm{~nm}$ is attributed to $\mathrm{n} \rightarrow \pi^{*}$ transition of the carbonyl groups $(\mathrm{C}=0$ bond $)[27,41]$.

\subsection{Validation of electrode modification}

We performed DPV scans to evaluate the effect of each nanomaterial and electrode modification used in our immunosensor (Fig. S2, Supplementary Material). Compared to peak current of $-14.59 \mu \mathrm{A}$ obtained with bare SPE, about three-fold increase in current (42.17 $\mu \mathrm{A}$ ) was obtained with PPy-SPE confirming the positive effect of PPy as a conducting polymer. The peak current further improved to -58.33 $\mu \mathrm{A}$ after AuNPs were deposited on PPy-SPE. Electrode modification with AuNPs not only improves the electrical conductivity but also serves to form Au-S bond for effective Ab immobilization. The apparent instability in conductivity and poor mechanical strength of PPy film is overcome by adding GO to form a PPy/GO nanocomposite, which also enhanced electron transfer with peak current of -80.2 
$\mu \mathrm{A}$, owing to partial reduction of $\mathrm{GO}$ in the process. Since $\mathrm{GO}$ is a poor conductor, it is usually fully reduced to rGO to improve its current response. However, when GO was electrochemically reduced in situ on PPy/GO-SPE to PPy/ErGO-SPE, the current signal was much better (-109.4 $\mu \mathrm{A})$ than when chemically reduced GO/PPy nanocomposite film was deposited on to SPE (i.e., with PPy/rGO-SPE). Thus we chose Ab-AuNPs-PPy/ErGO-SPE as the electrode for our investigation.

\subsection{Optimization of pyrrole concentration}

Figure 1 shows the $\mathrm{CV}$ responses of $5 \mathrm{mM}\left[\mathrm{Fe}(\mathrm{CN})_{6}\right]^{3-/ 4}$ in $1 \mathrm{M} \mathrm{KCl}$ at $\mathrm{SPEs}$ after PPy/GO electrochemical deposition using three different pyrrole concentrations $(0.025,0.05$, $0.1 \mathrm{M}$ ) with fixed concentration of $0.33 \mathrm{mg} / \mathrm{mL} \mathrm{GO}$ and $0.1 \mathrm{M} \mathrm{KCl}$. CV results show PPy/GO-SPE with $0.025 \mathrm{M}$ pyrrole concentration gives the characteristic well-defined sharp cathodic and anodic peaks with $160 \mathrm{mV}$ peak separations (Fig. 1a), which indicates very efficient electron transfer reaction between the electrolyte and the electrode surface. But, with increased pyrrole concentration $0.05 \mathrm{M}$ and $0.1 \mathrm{M}$, the peaks were less sharp and with greater separation (Figs. 1b and 1c), which demonstrates poor electron transfer. Hence, $0.025 \mathrm{M}$ pyrrole was determined as optimal.

\subsection{Electrochemical reduction of GO to ErGO}

For electrochemical reduction of GO, $\mathrm{CV}$ was performed in nitrogen-saturated $0.05 \mathrm{M}$ phosphate buffer $\left(\mathrm{Na}_{2} \mathrm{HPO}_{4} / \mathrm{NaH}_{2} \mathrm{PO}_{4}\right)$ solution ( $\mathrm{pH}$ 7.0) for 25 successive complete cycles in the potential range between 0 and $-1.4 \mathrm{~V}$ at the scan rate of $50 \mathrm{mV} / \mathrm{s}(\mathbf{F i g}$. S3, Supplementary Material). In this reduction step an irreversible peak at $-1.1 \mathrm{~V}$ was observed during the first cycle with an onset potential of $-0.65 \mathrm{~V}$, which is attributed to the reduction of oxygenated groups on GO surface $[35,42]$. The reduction current was greatly diminished in the 
subsequent cycles, and the peak vanished entirely after 25 cycles with stable current, which implies the complete reduction of GO.

\subsection{Depositing AuNPs on the working electrode surface}

We compared drop-coating and dip-coating methods to determine the effective way to deposit AuNPs on PPy/ErGO-SPE. DPV results in $5 \mathrm{mM}\left[\mathrm{Fe}(\mathrm{CN})_{6}\right]^{3-14-}$ in $1 \mathrm{M} \mathrm{KCl}$, showed sharper peak of about $180 \mu \mathrm{A}$ when AuNPs solution was drop-coated compared to very broad and small peak current when AuNPs solution was dip-coated (Fig. S4, Supplementary Material). Drop-coating of AuNPs yielded better electro-catalytic response perhaps due to more uniform dispersion of AuNPs on the PPy/ErGO surface than with dip-coating. Hence, the drop-coating method was selected.

\subsection{Optimization of antibody concentration}

We optimized the required anti-FB1 Ab and anti-DON Ab loading concentrations on the immunosensor for effective detection of FB1 and DON. The DPV responses at different anti-toxin Ab loads on AuNPs-PPy/ErGO-SPE were evaluated in the presence and absence of corresponding toxins containing $5 \mathrm{mM}\left[\mathrm{Fe}(\mathrm{CN})_{6}\right]^{3-/ 4-}$ in $1 \mathrm{M} \mathrm{KCl}$. The DPV results obtained with $40 \mu \mathrm{L}$ of different concentrations $(2,6,10,12 \mu \mathrm{g} / \mathrm{mL})$ of anti-FB1 Ab solution on four AuNPs-PPy/ErGO-SPE surfaces without (black line) and with $1 \mu \mathrm{g} / \mathrm{mL}$ of FB1 (red line) are presented in Fig. 2a. The current magnitude decreased initially with increasing Ab concentration and reached the lowest value at $10 \mu \mathrm{g} / \mathrm{mL}$ (black line). The red line further confirmed that the maximum current signal reduction after FB1 incubation occurs at 10 $\mu \mathrm{g} / \mathrm{mL} \mathrm{Ab}$ concentration, indicating the optimal formation of Ab-toxin complex and its subsequent insulating effect. Therefore, $40 \mu \mathrm{L}$ of $10 \mu \mathrm{g} / \mathrm{mL}$ anti-FB1 Ab loading (i.e., $0.4 \mu \mathrm{g}$ ) was selected as the optimal for FB1 detection. Similarly, DPV results obtained with $40 \mu \mathrm{L}$ of 
different concentrations of anti-DON Ab solution $(0.5,1,2,3 \mu \mathrm{g} / \mathrm{mL})$ are shown in Fig. $2 \mathrm{~b}$ in the absence (black line) and presence (red line) of $1 \mu \mathrm{g} / \mathrm{mL}$ DON. These results indicate that an anti-DON Ab loading of $40 \mu \mathrm{L}$ at $2 \mu \mathrm{g} / \mathrm{mL}$ is the optimal (i.e., $0.08 \mu \mathrm{g}$ ).

\subsection{Electrochemical and SEM characterization of fabricated SPE}

Figure 3A shows the $\mathrm{CV}$ results of $5 \mathrm{mM}\left[\mathrm{Fe}(\mathrm{CN})_{6}\right]^{3-/ 4-}$ in $1 \mathrm{M} \mathrm{KCl}$ at $50 \mathrm{mV} / \mathrm{s} \mathrm{scan}$ rate before and after each modification step on SPE working surface. With bare SPE (Fig. 3A, curve a), small peak value and large peak separation $(359 \mathrm{mV})$ reveals a quite slow electron transfer process. The redox peaks increased after the electrode was modified with PPy (Fig. 3A, curve b). After depositing PPy/GO film (Fig. 3A, curve c), both cathodic and anodic peaks further increased and much smaller peak separation $(160 \mathrm{mV})$ was observed, implying an improved electron transfer. However, it has been reported that oxygenated groups on GO could have insulating effects on the electrode and hinder electron transfer [43]. This can be resolved by removing the oxygenated groups on GO by reducing it to rGO, which was confirmed by the CV result of PPy/ErGO-SPE (Fig. 3A, curve d). Given the large surface area, excellent electrical conductivity and catalytic properties of AuNPs, the electrochemical response was further improved after AuNPs deposition with cathodic peak of $135 \mu \mathrm{A}$ (Fig. 3A, curve e), which is better than other reported electrodes [23, 39, 44]. A decrease in cathodic and anodic peaks obtained with Ab-AuNPs-PPy/ErGO-SPE (Fig. 3A, curve f) confirms that $\mathrm{Ab}$ was immobilized on the electrode surface effectively without denaturation.

The modified electrode was further characterized by electrochemical impedance spectroscopy (EIS). The Nyquist plots of impedance spectra in Fig. 3B show that after each SPE modification step the electron-transfer resistance $\left(\mathrm{R}_{\mathrm{et}}\right)$ changed, as observed by the diameter of the initial semicircle part of the plot. The $\mathrm{R}_{\mathrm{et}}$ for different modification steps 
determined by fitting to Randles equivalent circuit shown in Fig. 3B inset are as follow: bare SPE, 14 k $\Omega$; PPy-SPE, 12.2 k $\Omega$; PPy/GO-SPE, 9.9 k $\Omega$; PPy/ErGO-SPE, 1.83 k $\Omega$; AuNPsPPy/ErGO-SPE, $0.385 \Omega$; and Ab-AuNPs-PPy/ErGO-SPE, $3.10 \mathrm{k} \Omega$. The magnitude of $\mathrm{R}_{\mathrm{et}}$ is reflective of the dielectric and insulating properties of the working electrode. The larger the $\mathrm{R}_{\mathrm{et}}$, the worse the electron transfer ability. The changes in $\mathrm{R}_{\mathrm{et}}$ with each electrode modification step corroborate the CV results (Fig. 3A).

SEM images of the working electrode of SPEs after different modification steps are presented in Fig. 3C. The image of electrochemically deposited PPy film shows the uniform and microporous structure of PPy (Fig. 3C, image a), as it has been reported in the literature [27]. Similarly, the image of electrochemically grown PPy/GO film exhibits thin and wrapped GO sheet around PPy film with enhanced surface roughness and area (Fig. 3C, image b) $[45,46]$. After electrochemical reduction, the PPy/ErGO film shows more aggregated and rough surface of ErGO with occasional wrinkles and folds around PPy (Fig. 3C, image c) than reported methods [47, 48]. This may be attributed to the increased van der Waals attraction between adjacent layers due to the removal of oxygen functional groups, which confirms successful electrochemical reduction of GO [49, 50]. Fig. 3C, image d shows well distributed AuNPs on the PPy/ErGO film after drop-coating of AuNPs solution. These SEM images clearly indicate we have successfully fabricated well-integrated nanocomposite film with good orientation, conducting property and stability on the SPE working surface and obtained more intensive AuNPs deposition compared to published works [51, 52].

\subsection{Sensitivity, specificity, reproducibility and stability}

The DPV response of $5 \mathrm{mM}$ redox couple $\left[\mathrm{Fe}(\mathrm{CN})_{6}\right]^{3-14-}$ at as-prepared immunosensor was examined over a wide range of FB1 and DON concentrations in 1X PBS (Fig. 4A, B, blue lines). It was observed that, for both FB1 and DON, added toxin instantly 
reduced the magnitude of DPV peak current, and were inversely proportional to total toxin concentrations.

The LOD of the immunosensor was calculated using a linear regression curve based method reported in literature [53]:

$$
\mathrm{LOD}=3 * S_{\mathrm{a}} / b
$$

Where, $S_{\mathrm{a}}$ is the standard deviation of intercept and $b$ is the slope of the calibration curve. Using linear regressions presented in Fig. 4A and B (blue lines), we calculated LOD values for FB1 as $4.2 \mathrm{ppb}$ (linear range: 0.2 to $4.5 \mathrm{ppm}, \mathrm{R}^{2}=0.992$ ), and for DON it is $8.6 \mathrm{ppb}$ (linear range: 0.05 to $\left.1 \mathrm{ppm}, \mathrm{R}^{2}=0.994\right)$. As compared in Table 1, these LOD values are lower and were obtained over a wider linear range of toxin concentration than those obtained with other reported sensing techniques [21, 22, 24, 25, 39, 54-57].

The specificity of the immunosensor was tested by incubating it in target toxin solutions containing interfering agent. As shown in Fig. 4C, when the immunoelectrode was immobilized with anti-FB1 $\mathrm{Ab}$, the sensor responded only to $1 \mathrm{ppm}$ of $\mathrm{FB} 1$; the response for $1 \mathrm{ppm}$ of DON was the same as that obtained for blank 1X PBS solution. Furthermore, when equal amounts of DON (1 ppm) and FB1 (1 ppm) were mixed and tested, the DPV peak signal obtained was the same as that obtained for $1 \mathrm{ppm}$ of FB1 alone. Similar results were obtained for specificity towards DON using the immunoelectrode immobilized with antiDON Ab (Fig. 4D). These results indicate that our immunosensors are highly specific to the target toxin as long as the specific $\mathrm{Ab}$ is immobilized on the electrode surface.

The reproducibility of the immunosensor performance was evaluated by calculating relative standard deviation (\% RSD) values of three measurements made using three different Ab-AuNPs-PPy/ErGO-SPEs for $1 \mu \mathrm{g} / \mathrm{mL}$ of the toxins. The RSD values $5.7 \%$ and $4.9 \%$ were obtained for DON and FB1, respectively, confirm good reproducibility. 
The immunosensor stability was determined by measuring DPV current responses to DON after storing the electrode in $1 \mathrm{X}$ PBS at $4{ }^{\circ} \mathrm{C}$ for up to 12 days. Over the first six days the current signal remained fairly stable, changing very slightly from -82.1 to $-79.3 \mu \mathrm{A}$; however, somewhat larger decline from -79.3 to $-72.9 \mu \mathrm{A}$ was observed during the next six days 12 days (Fig. 5). Overall, an RSD of 5.8\% (n=3) obtained indicates good stability over 12 days.

\subsection{Sensor performance in extracts from spiked corn samples}

The calibration curves obtained for FB1 and DON in extracts obtained from spiked corn were plotted in Fig. 4A, B (red lines). The data from corn sample extracts are slightly lower than the corresponding results obtained when testing with buffer. However, both calibration curves showed similar trends, with even a slight increase in sensitivity, indicating negligible matrix effect. The recovery of spiked toxins in corn samples were in the range of $93.1 \%$ to $104.3 \%$ and their RSD values were less than $10 \%$ (Table 2). These results indicate very high acceptability of our sensor for detecting mycotoxin contamination in real food matrices.

\section{CONCLUSIONS}

We fabricated an electrochemical immunosensor using SPE for rapid and sensitive detection of two mycotoxins, FB1 and DON, using their respective anti-toxin Ab. The working electrode surface of SPE was modified with PPy, ErGO and AuNPs, which significantly improved electrochemical response and afforded effective Ab immobilization. The immunosensor was highly sensitive, with LOD of $4.2 \mathrm{ppb}$ for FB1 and $8.6 \mathrm{ppb}$ for DON, and its responses were linear and very reproducible (\%RSD<6\% for both FB1 and DON). The sensor performance, when tested with extracts obtained from toxin-spiked corn samples, 
exhibited high sensitivity and low matrix interference even in co-existing toxin environments. Hence, with specific antibodies, our sensing scheme is suitable for simultaneous detection of multiple co-contaminant mycotoxins individually.

\section{ACKNOWLEDGEMENT}

Work presented in this paper was supported by the USDA Hatch (WIS01644) and the Andersons Research Grants Program. The authors acknowledge the use of facilities and instrumentation supported by the UW MRSEC (DMR-1121288) and the UW NSEC (DMR0832760).

\section{REFERENCES}

[1] J.C. Frisvad, U. Thrane, Standardized high-performance liquid chromatography of 182 mycotoxins and other fungal metabolites based on alkylphenone retention indices and UVVIS spectra (diode array detection), J. Chromatogr. 404 (1987) 195-214.

[2] CAST, Mycotoxins: Risks in plant, animal, and human systems. Task Force Report No. 139. Council for Agricultural Science and Technology (CAST), Ames, Iowa, USA 2003.

[3] G.P. Anderson, V.A. Kowtha, C.R. Taitt, Detection of fumonisin b1 and ochratoxin a in grain products using microsphere-based fluid array immunoassays, Toxins (Basel) 2 (2010) 297-309.

[4] A. Arino, M. Herrera, T. Juan, G. Estopanan, Comparison of deoxynivalenol, ochratoxin $\mathrm{A}$ and aflatoxin B1 levels in conventional and organic durum semolina and the effect of milling, J. Food Nutr. Res. 48 (2009) 92-99.

[5] A. Pietri, M. Zanetti, T. Bertuzzi, Distribution of aflatoxins and fumonisins in dry-milled maize fractions, Food Addit. Contam. A 26 (2009) 372-380.

[6] NGFA, National Grain and Feed Association. FDA Mycotoxin Regulatory Guidance. https://www.ngfa.org/wp-content/uploads/NGFAComplianceGuideFDARegulatoryGuidanceforMycotoxins8-2011.pdf (Accessed March 28, 2016), 2011.

[7] F.S. Chu, G.Y. Li, Simultaneous occurrence of fumonisin B1 and other mycotoxins in moldy corn collected from the people's republic of china in regionswith high incidences of esophageal cancer., Appl. Environ. Microbiol. 60 (1994) 847-852.

[8] D.B. Carlson, D.E. Williams, J.M. Spitsbergen, P.F. Ross, C.W. Bacon, F.I. Meredith, R.T. Riley, Fumonisin B1 promotes aflatoxin B1 and N-methyl-N'-nitro-nitrosoguanidineinitiated liver tumors in rainbow trout, Toxicol. Appl. Pharmacol. 172 (2001) 29-36. 
[9] M. Castells, S. Marin, V. Sanchis, A.J. Ramos, Distribution of fumonisins and aflatoxins in corn fractions during industrial cornflake processing, Int. J. Food Microbiol. 123 (2008) $81-87$.

[10] M.G. Theumer, A.G. Lopez, M.P. Aoki, M.C. Canepa, H.R. Rubinstein, Subehronic mycotoxicoses in rats. Histopathological changes and modulation of the sphinganine to sphingosine ( $\mathrm{Sa} / \mathrm{So}$ ) ratio imbalance induced by Fusarium verticillioides culture material, due to the coexistence of aflatoxin B1 in the diet, Food. Chem. Toxicol. 46 (2008) 967-977.

[11] G. Sun, S. Wang, X. Hu, J. Su, Y. Zhang, Y. Xie, H. Zhang, L. Tang, J.S. Wang, Cocontamination of aflatoxin B-1 and fumonisin B-1 in food and human dietary exposure in three areas of China, Food Addit. Contam. A 28 (2011) 461-470.

[12] B. Birzele, A. Prange, J. Kramer, Deoxynivalenol and ochratoxin A in German wheat and changes of level in relation to storage parameters, Food Addit. Contam. 17 (2000) 10271035 .

[13] A. Hajjaji, M. El Otmani, D. Bouya, A. Bouseta, F. Mathieu, S. Collin, A. Lebrihi, Occurrence of mycotoxins (ochratoxin A, deoxynivalenol) and toxigenic fungi in Moroccan wheat grains: impact of ecological factors on the growth and ochratoxin A production, Mol. Nutr. Food Res. 50 (2006) 494-499.

[14] E.A. Dugan, The detection of aflatoxins by TLC, LC-GC North America 23 (2005) 51.

[15] S. Monbaliu, C. Van Poucke, C. Detavernier, F. Dumoulin, M. Van De Velde, E. Schoeters, S. Van Dyck, O. Averkieva, C. Van Peteghem, S. De Saeger, Occurrence of mycotoxins in feed as analyzed by a multi-mycotoxin LC-MS/MS method, J. Agric. Food Chem. 58 (2009) 66-71.

[16] C. Soares, P. Rodrigues, O. Freitas-Silva, L. Abrunhosa, A. Venancio, HPLC method for simultaneous detection of aflatoxins and cyclopiazonic acid, World Mycotoxin J. 3 (2010) $225-231$.

[17] X. Liu, Y. Xu, Q.H. He, Z.Y. He, Z.P. Xiong, Application of mimotope peptides of fumonisin b1 in Peptide ELISA, J. Agric. Food Chem. 61 (2013) 4765-4770.

[18] R. Ran, C. Wang, Z. Han, A. Wu, D. Zhang, J. Shi, Determination of deoxynivalenol (DON) and its derivatives: Current status of analytical methods, Food Control 34 (2013) $138-148$.

[19] N.A. Lee, S. Wang, R.D. Allan, I.R. Kennedy, A rapid aflatoxin B-1 ELISA: Development and validation with reduced matrix effects for peanuts, corn, pistachio, and soybeans, J. Agric. Food Chem. 52 (2004) 2746-2755.

[20] A.Y. Kolosova, W.B. Shim, Z.Y. Yang, S.A. Eremin, D.H. Chung, Direct competitive ELISA based on a monoclonal Ab for detection of aflatoxin B1. Stabilization of ELISA kit components and application to grain samples., Anal. Bioanal. Chem. 384 (2005) 286-294.

[21] M.K. Kadir, I.E. Tothill, Development of an electrochemical immunosensor for fumonisins detection in foods, Toxins (Basel) 2 (2010) 382-398. 
[22] A. Ezquerra, J.C. Vidal, L. Bonel, J.R. Castillo, A validated multi-channel electrochemical immunoassay for rapid fumonisin B1 determination in cereal samples., Anal. Methods 7 (2015) 3742-3749.

[23] S. Srivastava, V. Kumar, M.A. Ali, P.R. Solanki, A. Srivastava, G. Sumana, P.S. Saxena, A.G. Joshi, B.D. Malhotra, Electrophoretically deposited reduced graphene oxide platform for food toxin detection, Nanoscale 5 (2013) 3043-3051.

[24] D. Romanazzo, F. Ricci, G. Volpe, C.T. Elliott, S. Vesco, K. Kroeger, D. Moscone, J. Stroka, H. Van Egmond, M. Vehniainen, G. Palleschi, Development of a recombinant Fabfragment based electrochemical immunosensor for deoxynivalenol detection in food samples., Biosens. Bioelectron. 25 (2010) 2615-2621.

[25] W. Zhilei, S. Xiulan, L. Zaijun, F. Yinjun, R. Guoxiao, H. Yaru, L. Junkang, Highly sensitive deoxynivalenol immunosensor based on a glassy carbon electrode modified with a fullerene/ferrocene/ionic liquid composite, Microchim. Acta 172 (2011) 365-371.

[26] N. German, J. Voronovic, A. Ramanavicius, A. Ramanaviciene, Gold Nanoparticles and Polypyrrole for Glucose Biosensor Design, Procedia Eng. 47 (2012) 482-485.

[27] R. Seenivasan, W.J. Chang, S. Gunasekaran, Highly sensitive detection and removal of lead ions in water using cysteine-functionalized graphene oxide/polypyrrole nanocomposite film electrode, ACS Appl. Mater. Inter. 7 (2015) 15935-15943.

[28] T. Kuila, S. Bose, P. Khanra, A.K. Mishra, N.H. Kim, J.H. Lee, Recent advances in graphene-based biosensors, Biosens. Bioelectron. 26 (2011) 4637-4648.

[29] M. Pumera, Graphene in biosensing, Mater. Today 14 (2011) 308-315.

[30] Y.C. Wang, S. Gunasekaran, Spectroscopic and microscopic investigation of gold nanoparticle nucleation and growth mechanisms using gelatin as a stabilizer, J. Nanopart. Res. 14 (2012) 1-11.

[31] J. Guan, Y.C. Wang, S. Gunasekaran, Using l-arginine-functionalized gold nanorods for visible detection of mercury(II) ions, J. Food Sci. 80 (2015) N828-N833.

[32] P.O. Bagci, Y.C. Wang, S. Gunasekaran, A simple and green route for room-temperature synthesis of gold nanoparticles and selective colorimetric detection of cysteine, J. Food Sci. 80 (2015) N2071-N2078.

[33] J. Zhong, S. Gao, G. Xue, B. Wang, Study on Enhancement Mechanism of Conductivity Induced by Graphene Oxide for Polypyrrole Nanocomposites, Macromolecules 48 (2015) $1592-1597$.

[34] X. Xu, D. Huang, K. Cao, M. Wang, S.M. Zakeeruddin, M. Gratzel, Electrochemically reduced graphene oxide multilayer films as efficient counter electrode for dye-sensitized solar cells, Sci. Rep. 3 (2013) 1489.

[35] H.L. Guo, X.F. Wang, Q.Y. Qian, F.B. Wang, X.H. Xia, A Green Approach to the Synthesis of Graphene Nanosheets, ACS Nano 3 (2009) 2653-2659. 
[36] F. Wang, J. Wang, H. Chen, S. Dong, Assembly process of CuHCF/MPA multilayers on gold nanoparticles modified electrode and characterization by electrochemical SPR, J. Electroanal. Chem. 600 (2007) 265-274.

[37] C. Yi, S. Qi, D. Zhang, M. Yang, Covalent conjugation of multi-walled carbon nanotubes with proteins, Methods Mol. Biol. 625 (2010) 9-17.

[38] R. Seenivasan, N. Maddodi, V. Setaluri, S. Gunasekaran, An electrochemical immunosensing method for detecting melanoma cells, Biosens. Bioelectron. 68 (2015) 508515.

[39] C.E. Sunday, M. Masikini, L. Wilson, C. Rassie, T. Waryo, P.G. Baker, E.I. Iwuoha, Application on gold nanoparticles-dotted 4-nitrophenylazo graphene in a label-free impedimetric deoxynivalenol immunosensor, Sensors (Basel) 15 (2015) 3854-3871.

[40] X. Wang, W. Xing, B. Yu, X. Feng, L. Song, Y. Hu, A facile and cost-effective approach to the reduction of exfoliated graphite oxide using sodium hypophosphite under acidic conditions, J. Mater. Chem. C 1 (2013) 690-694.

[41] Z. Luo, Y. Lu, L.A. Somers, A.T. Johnson, High yield preparation of macroscopic graphene oxide membranes, J. Am. Chem. Soc. 131 (2009) 898-899.

[42] B. Unnikrishnan, S. Palanisamy, S.M. Chen, A simple electrochemical approach to fabricate a glucose biosensor based on graphene-glucose oxidase biocomposite, Biosens. Bioelectron. 39 (2013) 70-75.

[43] I. Jung, D.A. Dikin, R.D. Piner, R.S. Ruoff, Tunable electrical conductivity of individual graphene oxide sheets reduced at "low" temperatures, Nano Lett. 8 (2008) 4283-4287.

[44] M. Chen, C. Zhao, W. Chen, S. Weng, A. Liu, Q. Liu, Z. Zheng, J. Lin, X. Lin, Sensitive electrochemical immunoassay of metallothionein-3 based on $\mathrm{K} 3[\mathrm{Fe}(\mathrm{CN}) 6]$ as a redox-active signal and C-dots/Nafion film for Ab immobilization, Analyst 138 (2013) 7341-7346.

[45] J. Li, H. Xie, Y. Li, Fabrication of graphene oxide/polypyrrole nanowire composite for high performance supercapacitor electrodes, J. Power Sources 241 (2013) 388-395.

[46] C. Zhu, J. Zhai, D. Wen, S. Dong, Graphene oxide/polypyrrole nanocomposites: onestep electrochemical doping, coating and synergistic effect for energy storage, J. Mater. Chem. 22 (2012) 6300-6306.

[47] M. Wang, W. Yuan, X. Yu, G. Shi, Picomolar detection of mercury (II) using a threedimensional porous graphene/polypyrrole composite electrode, Anal. Bioanal. Chem. 406 (2014) 6953-6956.

[48] P. Si, H. Chen, P. Kannan, D.-H. Kim, Selective and sensitive determination of dopamine by composites of polypyrrole and graphene modified electrodes, Analyst 136 (2011) 5134-5138.

[49] H.D. Yoo, J.H. Jang, K. Cho, Y. Zheng, Y. Park, J.H. Ryu, S.M. Oh, Effects of Interlayer Distance and van der Waals Energy on Electrochemical Activation of Partially Reduced Graphite Oxide, Electrochim. Acta 173 (2015) 827-833. 
[50] < Few-layer Graphene Prepared Via Microwave Digestion Reduction and its Electrochemical Performances in Lithium Ion Batteries.pdf $>$.

[51] T. Qian, C. Yu, X. Zhou, S. Wu, J. Shen, Au nanoparticles decorated polypyrrole/reduced graphene oxide hybrid sheets for ultrasensitive dopamine detection, Sensor Actuat. B-Chem. 193 (2014) 759-763.

[52] Y. Yang, A.M. Asiri, D. Du, Y. Lin, Acetylcholinesterase biosensor based on a gold nanoparticle-polypyrrole-reduced graphene oxide nanocomposite modified electrode for the amperometric detection of organophosphorus pesticides, Analyst 139 (2014) 3055-3060.

[53] A. Shrivastava, V.B. Gupta, Methods for the determination of limit of detection and limit of quantitation of the analytical methods, Chron. Young Sci. 2 (2011) 21-25

[54] F. Ricci, P. Flavio, M. Abagnale, M. Messia, E. Marconi, G. Volpe, D. Moscone, G. Palleschi, Direct electrochemical detection of trichothecenes in wheat samples using a 96well electrochemical plate coupled with microwave hydrolysis, World Mycotoxin J. 2 (2009) 239-245.

[55] C.X. Dominguez, R.A. Amezquita, T.X. Guan, H.D. Marshall, N.S. Joshi, S.H. Kleinstein, S.M. Kaech, The transcription factors ZEB2 and T-bet cooperate to program cytotoxic T cell terminal differentiation in response to LCMV viral infection, J. Exp. Med. 212 (2015) 2041-2056.

[56] A. Jodra, M.A. Lopez, A. Escarpa, Disposable and reliable electrochemical magnetoimmunosensor for Fumonisins simplified determination in maize-based foodstuffs, Biosens. Bioelectron. 64 (2015) 633-638.

[57] X. Yang, X. Zhou, X. Zhang, Y. Qing, M. Luo, X. Liu, C. Li, Y. Li, H. Xia, J. Qiu, A Highly Sensitive Electrochemical Immunosensor for Fumonisin B1Detection in Corn Using Single-Walled Carbon Nanotubes/Chitosan, Electroanal. 27 (2015) 2679-2687.

[58] W. Gu, P. Zhu, D. Jiang, X. He, Y. Li, J. Ji, L. Zhang, Y. Sun, X. Sun, A novel and simple cell-based electrochemical impedance biosensor for evaluating the combined toxicity of DON and ZEN., Biosens. Bioelectron. 70 (2015) 447-454. 


\section{SCHEME AND FIGURES}

(A)

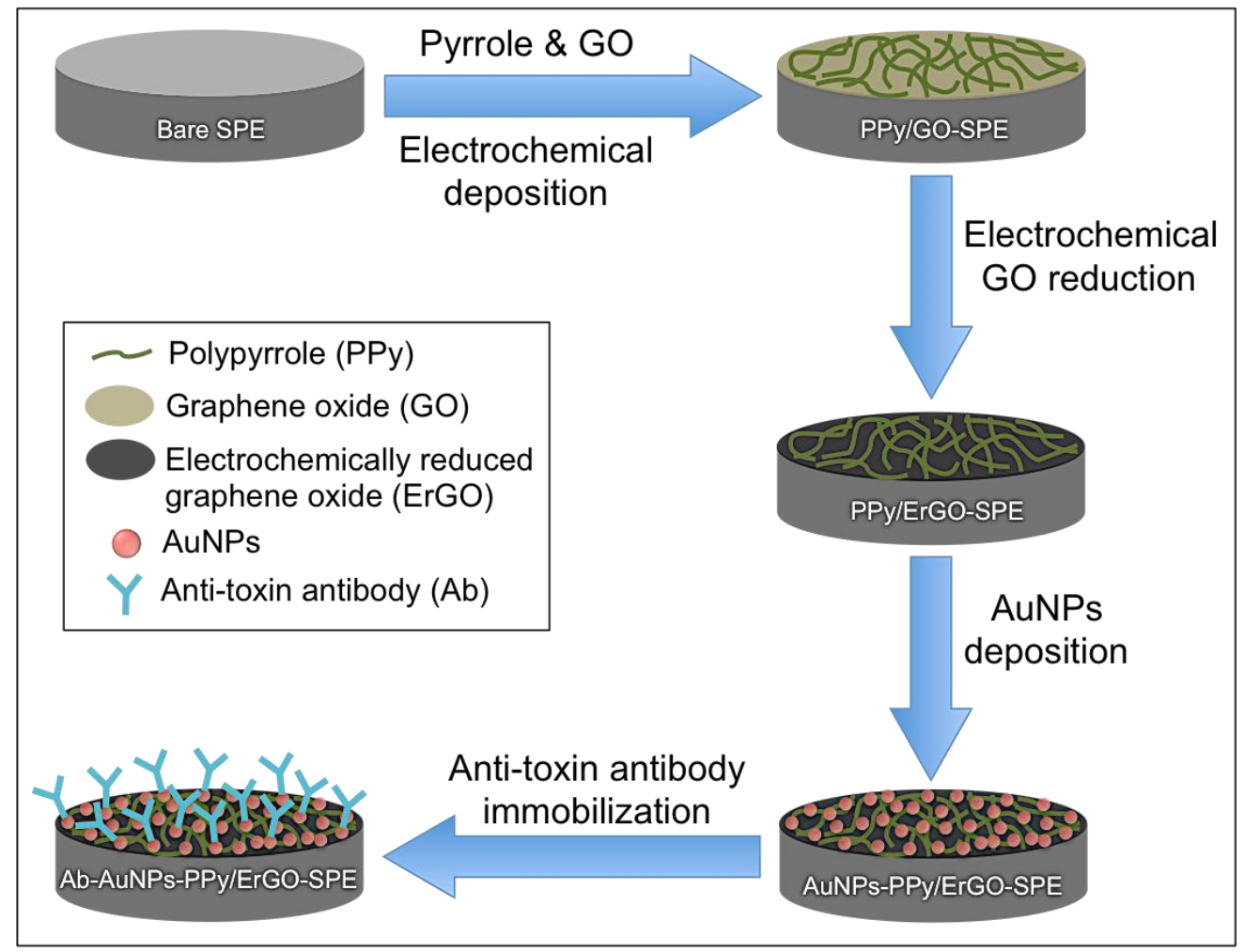

(B)

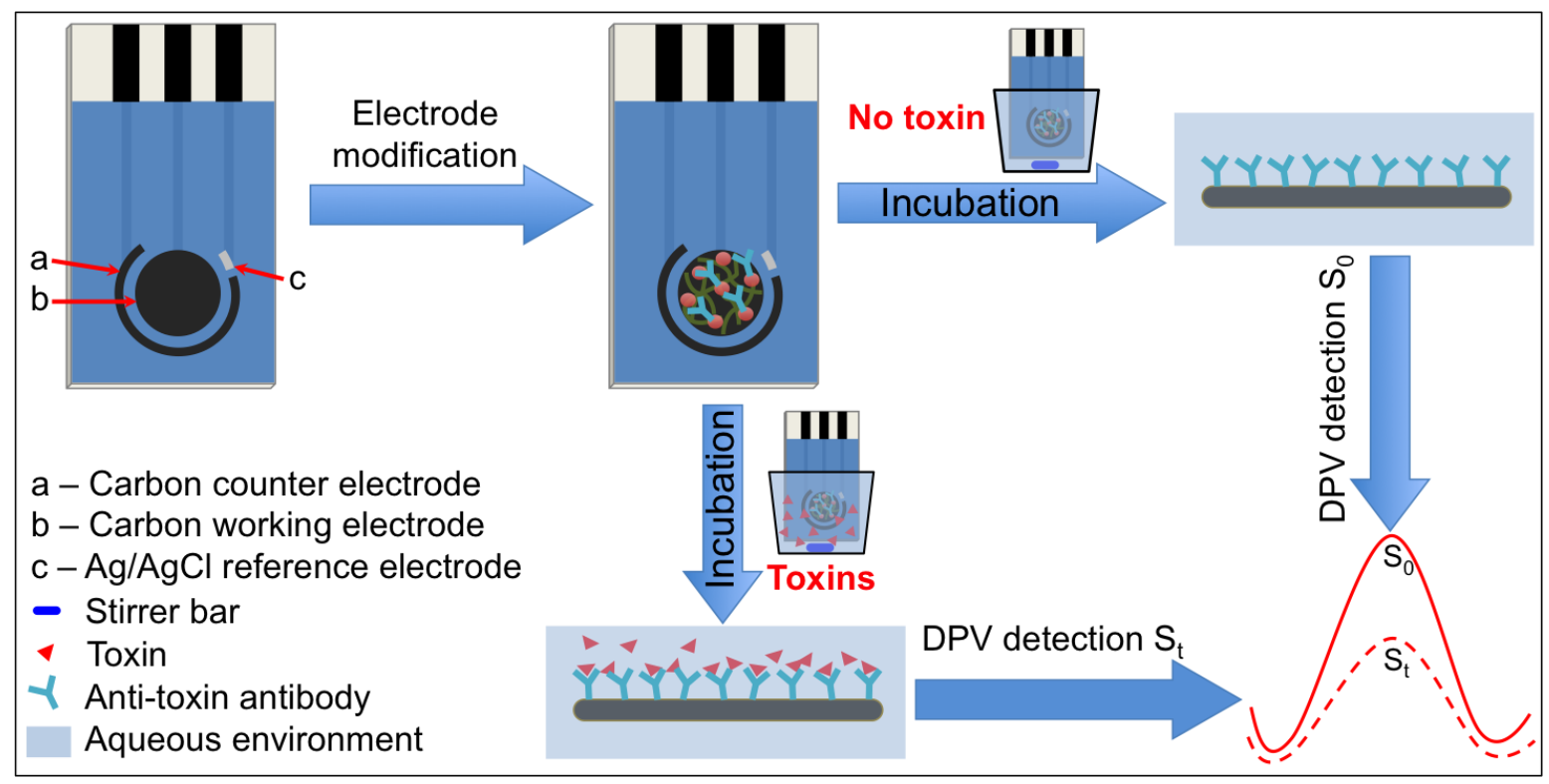

Scheme 1. (A) Illustration of step-by-step fabrication of the immunosensor and (B) electrochemical immunosensing employed for the detection of mycotoxins. 

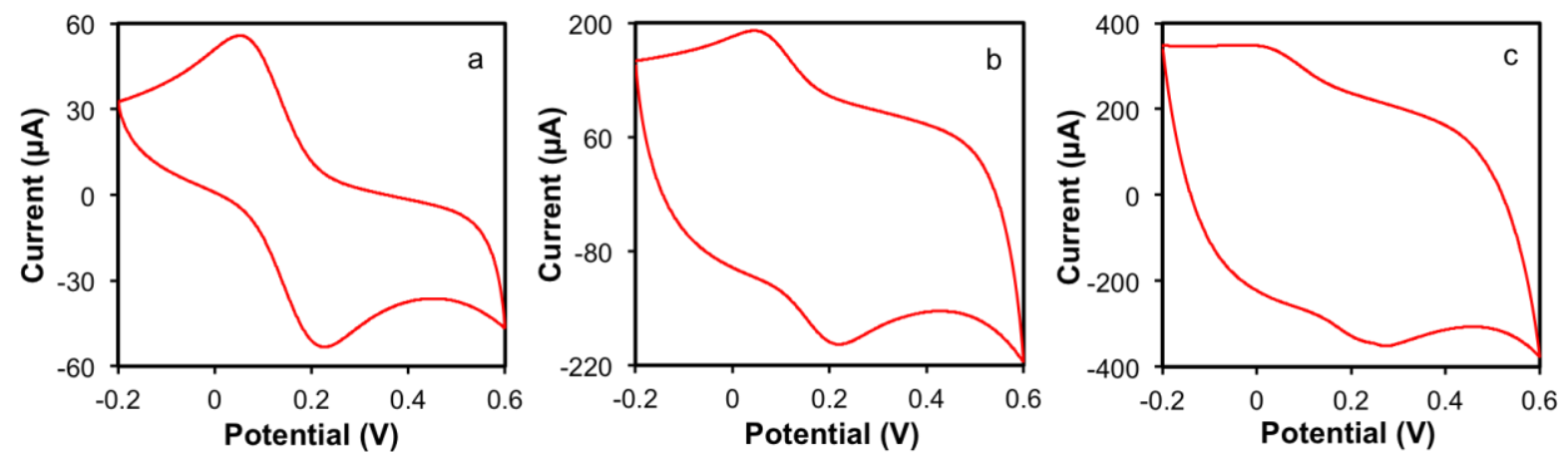

Fig. 1. $\mathrm{CV}$ responses of $5 \mathrm{mM}\left[\mathrm{Fe}(\mathrm{CN})_{6}\right]^{3-14-}$ in $1 \mathrm{M} \mathrm{KCl}$ at PPy/GO electrochemically deposited on SPE at pyrrole concentrations of (a) $0.025 \mathrm{M}$, (b) $0.05 \mathrm{M}$ and (c) $0.1 \mathrm{M}$. GO concentration $=0.33 \mathrm{mg} / \mathrm{mL} ; \mathrm{KCl}$ concentration $=0.1 \mathrm{M}$. 

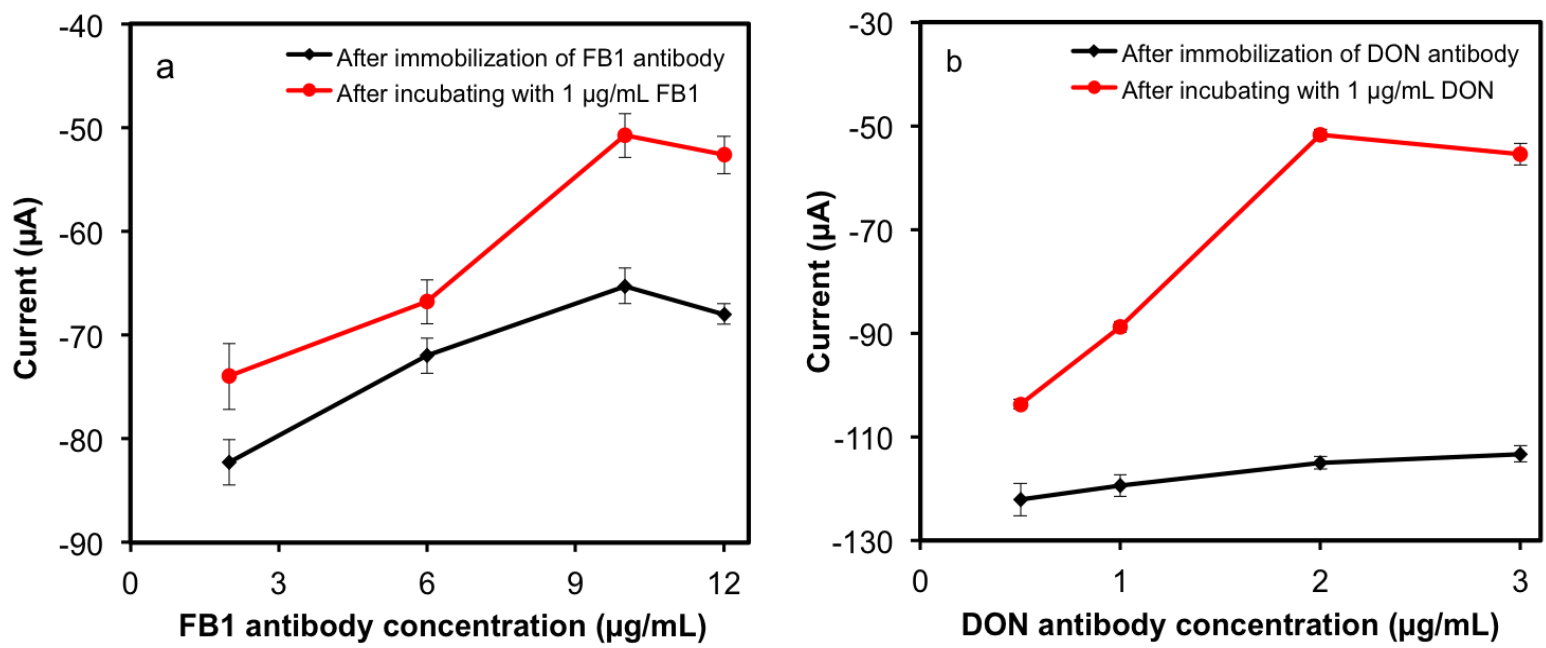

Fig. 2. DPV responses for (a) anti-FB1 Ab and FB1 (b) anti-DON Ab and DON. Incubation period with FB1 or DON = 40 min; values are means of four independent measurements, and error bars are standard deviations. 

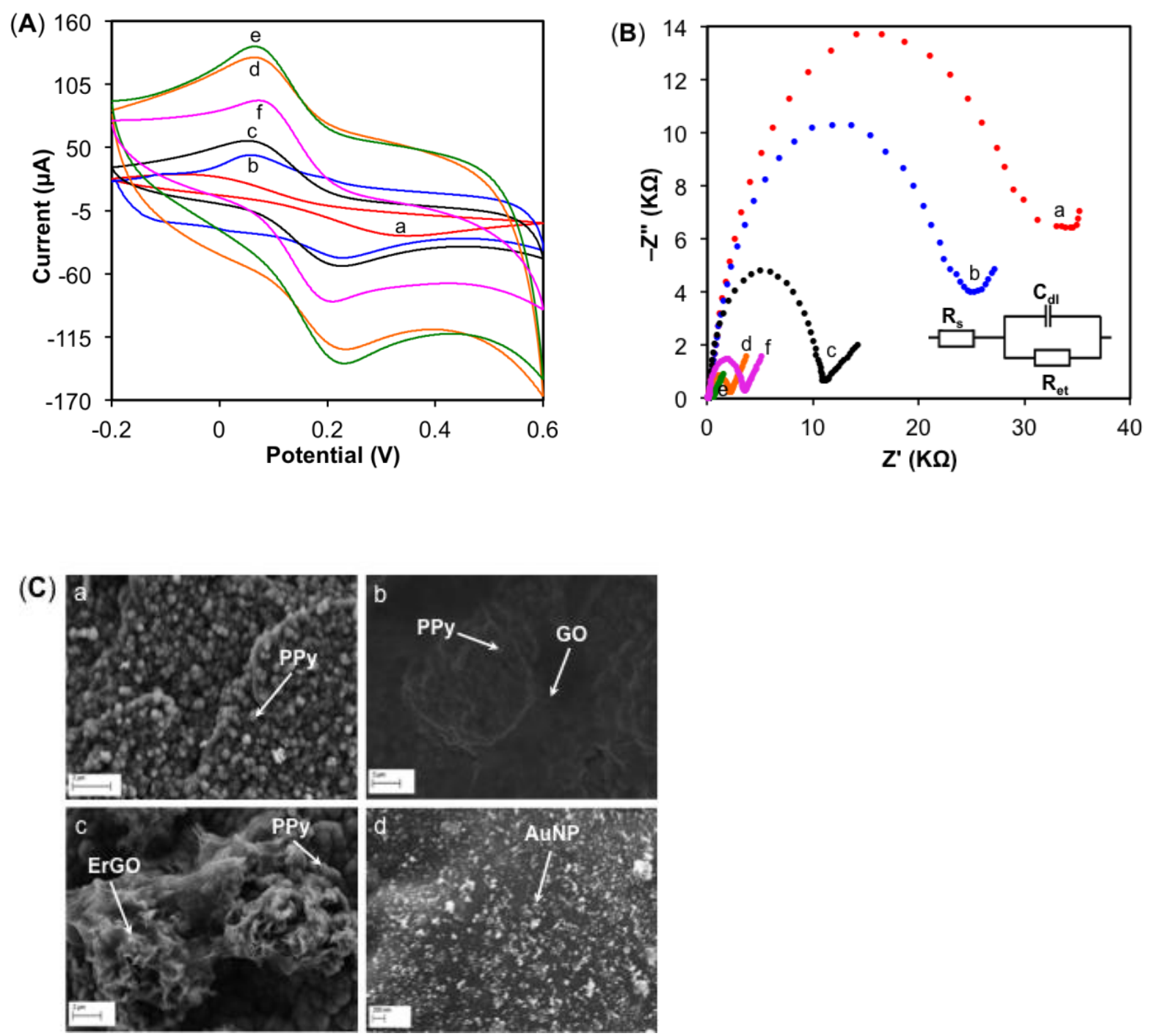

Fig. 3. (A) $\mathrm{CV}$ and (B) EIS responses of $5 \mathrm{mM}\left[\mathrm{Fe}(\mathrm{CN})_{6}\right]^{3-/ 4-}$ in $1 \mathrm{M} \mathrm{KCl}$ at (a) bare $\mathrm{SPE}$, (b) PPy-SPE, (c) PPy/GO-SPE, (d) PPy/ErGO-SPE, (e) AuNPs-PPy/ErGO-SPE and (f) AbAuNPs-PPy/ErGO-SPE. CV scan rate $=50 \mathrm{mV} / \mathrm{s}$ and applied potential range $=-0.2$ to $+0.6 \mathrm{~V}$. In Randles equivalent circuit ( $B$ inset), $R_{s}$, electrolyte resistance; $R_{\mathrm{et}}$, electron-transfer resistance; $\mathrm{C}_{\mathrm{dl}}$, double layer capacitance. EIS frequency range $=0.1$ to $100,000 \mathrm{~Hz}$ and amplitude $=5 \mathrm{mV}$. (C) SEM images of working electrode surface of SPE modified with (a) PPy (b) PPy/GO (c) PPy/ErGO and (d) AuNPs-PPy/ErGO. 

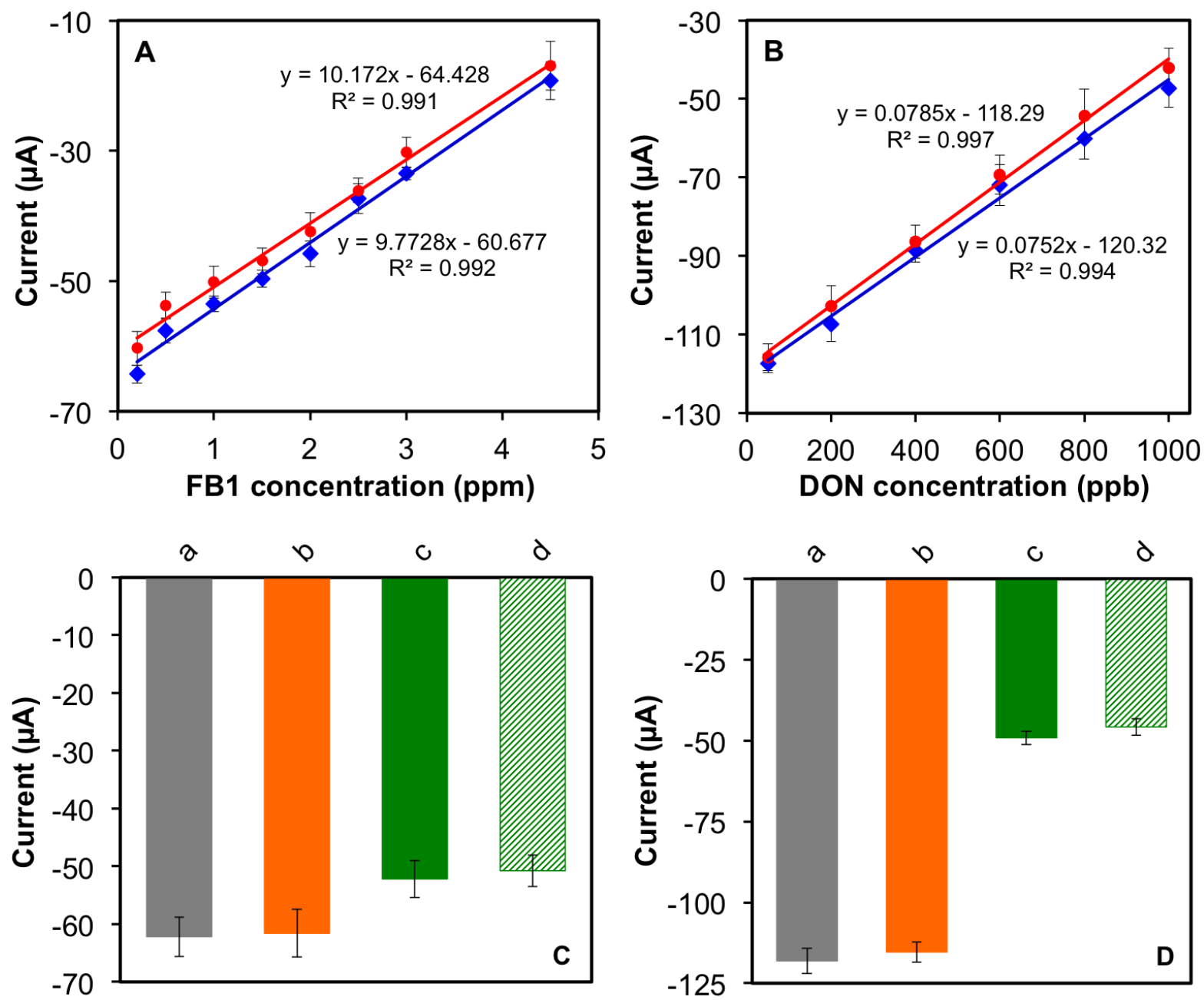

Fig. 4. Linear calibration plots of DPV peak current measured (at applied potential $=-0.2$ to +0.3 V) using our immunosensor (Ab-AuNPs-PPy/ErGO-SPE) for (A) 0.2 to 4.5 ppm FB1 with $0.4 \mu \mathrm{g}$ anti-FB1 Ab in buffer (blue) and corn extract (red), and (B) 0.05 to 1 ppm DON with $0.08 \mu \mathrm{g}$ anti-DON Ab in buffer (blue) and corn extract (red). Values are mean of three independent measurements, and error bars are standard deviations. Specificity of (C) FB1 immunosensor against (a) PBS 0 ppm FB1, (b) 1 ppm DON, (c) 1 ppm FB1 and (d) 1 ppm FB1 + 1 ppm DON, and (D) DON immunosensor against (a) PBS 0 ppm DON, (b) 1 ppm FB1, (c) 1 ppm DON and (d) 1 ppm DON + 1 ppm FB1 under optimal experimental conditions. Error bars are standard deviations of three independent measurements. 


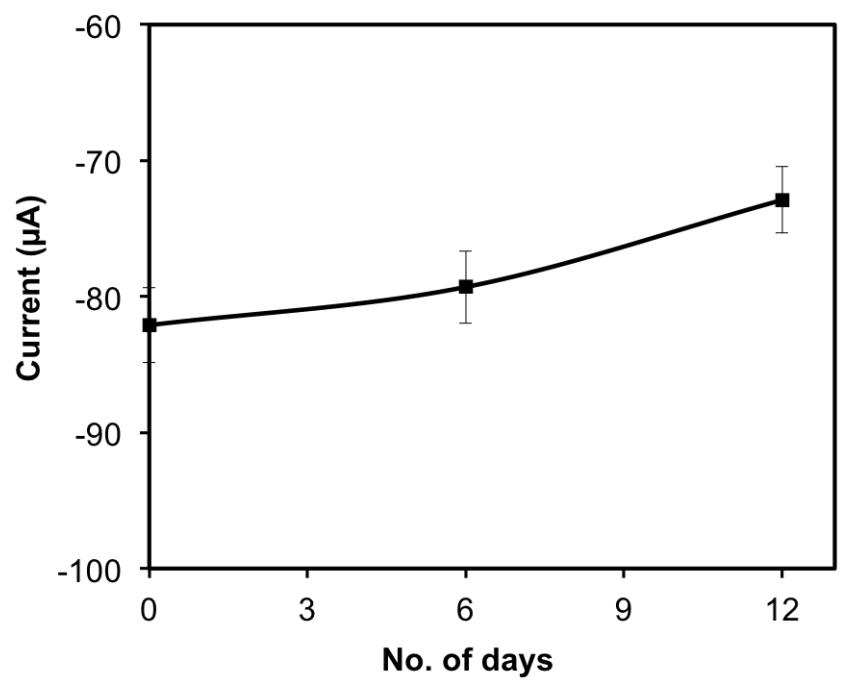

Fig. 5. Stability of the immunosensor stored in $1 \mathrm{X}$ PBS at $4{ }^{\circ} \mathrm{C}$ over 12 days. Error bars are standard deviations of three independent measurements. 
Table 1. Comparison of the sensing characteristics of our immunosensor (Ab-AuNPsPPy/ErGO-SPE) and those reported in literature

\begin{tabular}{|c|c|c|c|c|}
\hline Mycotoxin & Sensor format & Linear range & $\begin{array}{l}\text { Limit of } \\
\text { detection }\end{array}$ & Reference \\
\hline DON & ELIME assay & $\begin{array}{l}100-4500 \\
\mathrm{ng} / \mathrm{mL}\end{array}$ & $63 \mathrm{ng} / \mathrm{mL}$ & [24] \\
\hline $\mathrm{DON}$ & $\begin{array}{l}\text { Label-free inhibition based } \\
\text { electrochemical } \\
\text { immunosensor }\end{array}$ & $6-30 \mathrm{ng} / \mathrm{mL}$ & $0.3 \mu \mathrm{g} / \mathrm{mL}$ & [39] \\
\hline DON & 96-well SPE plate & $2-20 \mu \mathrm{g} / \mathrm{g}$ & $1.1 \mu \mathrm{g} / \mathrm{g}$ & {$[54]$} \\
\hline DON & $\begin{array}{l}\text { AuNPs/p- } \\
\text { aminothiophenol/folic } \\
\text { acid/GC }\end{array}$ & $0.1-20 \mu \mathrm{g} / \mathrm{mL}$ & $0.03 \mu \mathrm{g} / \mathrm{mL}$ & {$[58]$} \\
\hline DON & Ab-AuNPs-PPy/ErGO-SPE & $0.05-1 \mathrm{ppm}$ & $8.6 \mathrm{ppb}$ & Our sensor \\
\hline $\begin{array}{l}\mathrm{FB} 1+\mathrm{FB} 2 \\
+\mathrm{FB} 3\end{array}$ & $\begin{array}{l}\text { Electrochemical } \\
\text { magnetoimmunosensor }\end{array}$ & & $0.33 \mu \mathrm{g} / \mathrm{L}$ & {$[56]$} \\
\hline $\mathrm{FB} 1+\mathrm{FB} 2$ & $\begin{array}{l}\text { Direct } \\
\text { competitive/Amperometry }\end{array}$ & $1-1000 \mathrm{ng} / \mathrm{mL}$ & $5 \mathrm{ng} / \mathrm{mL}$ & {$[21]$} \\
\hline FB1 & $\begin{array}{l}\text { Multi-channel } \\
\text { electrochemical sensor }\end{array}$ & $0-54 \mu \mathrm{g} / \mathrm{L}$ & $0.58 \mu \mathrm{g} / \mathrm{L}$ & {$[22]$} \\
\hline FB1 & $\begin{array}{l}\text { Electrochemical } \\
\text { immunosensor }\end{array}$ & $\begin{array}{l}0.01-1000 \\
\mathrm{ng} / \mathrm{mL}\end{array}$ & $0.002 \mathrm{ng} / \mathrm{mL}$ & {$[57]$} \\
\hline FB1 & Ab-AuNPs-PPy/ErGO-SPE & $0.2-4.5 \mathrm{ppm}$ & $4.2 \mathrm{ppb}$ & Our sensor \\
\hline
\end{tabular}

Abbreviations: DON: deoxynivaleno, FB1: fumonisin B1, FB2: fumonisin B2, AuNPs: gold nanoparticles, GC: glassy carbon, ELIME: Enzyme-Linked-ImmunomagneticElectrochemical, SPE: screen printed electrode, EIS: electrochemical impedance spectroscopy, Ab: Ab, PPy: polypyrrole, ErGO: electrochemically reduced graphene oxide. 
Table 2. Recovery of FB1 and DON from spiked corn samples when tested using extracts.

\begin{tabular}{ccccc}
\hline Toxin & $\begin{array}{c}\text { Spiked } \\
(\boldsymbol{\mu g} / \mathbf{k g})\end{array}$ & $\begin{array}{c}\text { Measured } \\
(\boldsymbol{\mu g} / \mathbf{k g})\end{array}$ & $\begin{array}{c}\text { RSD } \\
(\boldsymbol{\%})\end{array}$ & $\begin{array}{c}\text { Recovery } \\
(\%)\end{array}$ \\
\hline & 100 & 94 & 8.51 & 94.1 \\
FB1 & 500 & 484.9 & 3.30 & 97.0 \\
& 2000 & 1904.2 & 2.21 & 95.2 \\
& 4000 & 4141.6 & 1.71 & 103.5 \\
\hline \multirow{2}{*}{ DON } & 100 & 93.1 & 9.76 & 93.1 \\
& 500 & 521.7 & 4.0 & 104.3 \\
\hline
\end{tabular}

